\title{
A nano-view of movement in cells
}

\section{Methods to label proteins with carbon nanotubes permit the study of intracel- lular movement at different timescales.}

To assert that the inside of a cell is dynamic is a truism. But what may be easy to say is not necessarily easy to study. Understanding movement within cells requires methods that can interrogate dynamics over a wide range of temporal and spatial scales. In a recent paper, an interdisciplinary group of researchers at the junction of biophysics, theory and nanotechnology reports the development of such a method.

Following her doctoral work on the properties of single-walled carbon nanotubes (SWNTs) in the laboratory of Matteo Pasquali at Rice University, first author Nikta Fakhri began applying these nanotubes to track proteins within cells, now in the laboratory of Christoph Schmidt at the University of Georg-August-Universität Göttingen. SWNTs are carbon nanostructures about 1 nanometer $(\mathrm{nm})$ in diameter and of varying length that fluoresce in the infrared (900-1,400 $\mathrm{nm}$ ), a region of the electromagnetic spectrum in which biological samples have almost no autofluorescence. SWNTs do not bleach or blink, and they have short fluorescence lifetimes, meaning that they can in principle be imaged for long periods, with high signalto-noise ratio and flexible temporal resolution.

The key to using these probes to monitor intracellular dynamics, explains Fred MacKintosh at the Vrije Universiteit, the theorist on the team, was to find a way to specifically label a particular protein within the cell. To do this, Fakhri and colleagues had to first maintain the SWNTs in a dispersed state in the intracellular milieu in such a way that the cell was unharmed and nanotube fluorescence remained stable. They achieved this by wrapping the tubes in single-stranded DNA. Then, after some trial and error, the researchers settled on HaloTag to covalently

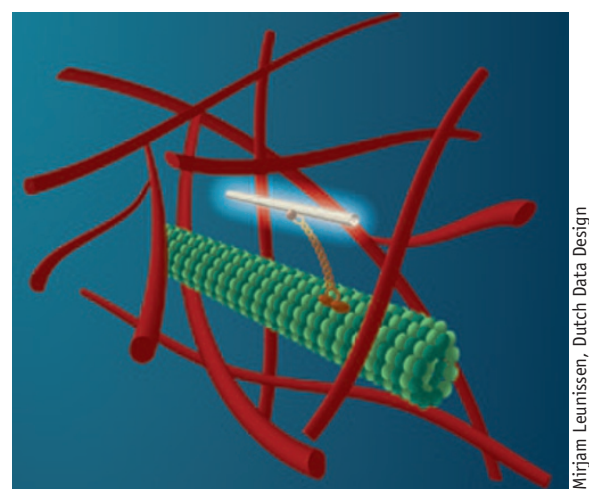

Using carbon nanotubes to follow molecular motion. Microtubules are in green, the motor is in yellow and actin filaments are in red.

couple the SWNTs to the molecular motor kinesin. Other researchers will probably not need extensive reoptimization to adapt this approach to their own work, Schmidt believes. "All the steps that had to be tried and tested are now taken, and it is highly likely that a similar approach is going to work for most any protein one might want to target in cells," he says.

The same may not be true for potential toxic effects of these nanoparticles. Though chemical toxicity is unlikely for these inert, all-carbon structures, and although Fakhri and colleagues observed no ill effects on COS cells, it would be prudent to reexamine toxicity in any system in which the approach is used. Similarly, although tagging and labeling with $100-$ to $300-\mathrm{nm}$ long SWNTs did not alter kinesin behavior (the researchers report directed molecular movements in keeping with what is already known about the molecule's function), this should be assessed anew for every target protein.

Upon electroporation of the wrapped SWNTs into COS cells expressing Halotagged kinesin, the researchers observed that a substantial fraction of the nanotubes (30\%) displayed directed motion, with the rest moving randomly but in a locally constrained fashion. Most of the intracellular nanotubes are likely to be attached to kinesin, Schmidt predicts because, with about 100 nanotubes per cell, the researchers are working at a large excess of motors compared to probes. By focusing on nanotubes that initially displayed random movement that subsequently became directed, the researchers could be sure they were monitoring attached probes.

The exceptional properties of SWNTs meant that they could be tracked within cells for as long as 1.5 hours, at a resolution of tens of nanometers, and over timescales spanning five orders of magnitude. As a consequence, not only could the researchers monitor single kinesin molecules moving along cellular microtubules-a difficult feat but one that has been achieved before-but they could probe other nonequilibrium intracellular movements too. Some of the seemingly random movements of the nanotubes, they realized, contained interesting information. "This is where the theory part of the collaboration is powerful," says MacKintosh.

At timescales intermediate between fast thermal motion and (relatively) slow directed kinesin tracking along microtubules, the researchers observed movements of the probes transverse to the microtubule tracks that they were able to attribute, on the basis of a theoretical model, to nonthermal movements of the microtubules themselves; furthermore, they identified the underlying cause of these movements to be myosin-driven processes. With this elegant use of nanotubes, a picture thus begins to emerge of a very mobile cytoplasm in which motors and their cargo move along the cytoskeleton and in which active, motordriven nondirected movements constantly buffet intracellular structures as well.

\section{Natalie de Souza}

\section{RESEARCH PAPERS}

Fakhri, N. et al. High-resolution mapping of intracellular fluctuations using carbon nanotubes. Science 344, 1031-1035 (2014). 